\title{
Painlevé IV and degenerate Gaussian Unitary Ensembles
}

\author{
Yang Chen ${ }^{\dagger}$ \\ Department of Mathematics \\ Imperial College London \\ 180 Queen's Gates \\ London SW7 2BZ UK
}

\author{
M. V. Feigin* \\ Department of Mathematics \\ University Glasgow \\ University Gardens \\ Glasgow G12 8QW UK
}

28-06-2006

\begin{abstract}
We consider those Gaussian Unitary Ensembles where the eigenvalues have prescribed multiplicities, and obtain joint probability density for the eigenvalues. In the simplest case where there is only one multiple eigenvalue $t$, this leads to orthogonal polynomials with the Hermite weight perturbed by a factor that has a multiple zero at $t$. We show through a pair of ladder operators, that the diagonal recurrence coefficients satisfy a particular Painlevé IV equation for any real multiplicity. If the multiplicity is even they are expressed in terms of the generalized Hermite polynomials, with $t$ as the independent variable.

*m.feigin@maths.gla.ac.uk
\end{abstract}

$\dagger$ ychen@ic.ac.uk 


\section{Introduction}

Random matrix ensembles originally conceived to explain the statistical properties of the energy levels in heavy nuclei 30] has recently seen applications in transport in disordered systems, string theory and various areas of pure and applied mathematics. In addition to classical quantities of interest such as the correlation functions, the average of the product of characteristic polynomials of random matrices were under investigation starting from Brezin-Hikami paper [5] (see also [4] and references therein).

From the Painlevé equations point of view the average of a power of characteristic polynomial in Gaussian unitary ensemble gives a $\tau$-function of the rational solution of Painleve IV equation. For the integer powers this can be seen from the original Kajiwara-Ohta determinant formula for the rational solutions of PIV ([16], c.f. [22]) and it was later explored by Forrester and Witte [13].

In this paper we consider the degenerate gaussian unitary ensembles. That is we restrict ourselves to the nonlinear subspace of Hermitian matrices having prescribed spectrum degeneracy. Various statistical properties on eigenvalues of such matrices can be asked. The first natural question we answer is the determination of the joint probability density of the eigenvalues when these have some multiplicity. It happens that as in the case of classical ensembles (see [20]) the joint probability density has the form of product of pairwise differences between the different eigenvalues taken in the powers depending on the multiplicities. Thus we naturally arrive to considering orthogonal polynomials with Hermite weight perturbed (multiplied) by a product of linear factors.

These types of weights also appear in the random matrix theory in consideration of the averages of characteristic polynomials (see [5], [2]), although in that case the zeroes of these factors are the external variables to the matrices of the ensembles. We note that an orthogonal circular random matrix ensemble with fixed degenerate eigenvalue at 1 was considered by Snaith in [26] in conjectural relation to number theoretic questions on $L$ functions of elliptic curves. More general Jacobi circular ensembles were studied recently in [1]. A general approach to the joint probability density of ensembles of various type was suggested recently in [1].

We are also motivated by the theory of Calogero-Moser-Sutherland systems. The ground states of these systems at appropriate interaction parameter coincide with the joint probability densities of eigenvalues in the classical ensembles. The joint probability density for degenerate ensembles coincides with the factorized wave function for the appropriate multi-species generalisation of Calogero-Moser problem considered in [12. This type of generalisation is integrable in the case of two types of particles [6], 24]. More remarkably, Sergeev and Veselov showed that the corresponding quantum Hamiltonian can be obtained by applying a restriction procedure on the Calogero-Moser-Sutherland Hamiltonian in the infinite dimensional space to the appropriate discriminant [25]. We plan to elaborate these relations in future.

In the context of orthogonal polynomials, perturbations of the standard weights such as the Jacobi weight by special factors is an important topic of investigation, where the problem 
is the determination of the recurrence coefficients from the weights (see [19, [21] and the references therein). In particular, it was noted by Magnus in [18 that often the variations lead to the recurrence coefficients which are solutions to the nonlinear equations. In some cases the appearance of Painlevé IV for the certain exponential weights was established 18. More recently, it was shown in [10] that the diagonal recurrence coefficient associated with the Hermite weight perturbed by special discontinuous factor satisfies a particular Painlevé IV.

In this paper we show that when the Hermite weight is perturbed by a linear factor having multiple zero the diagonal recurrence coefficients satisfy a particular two parameter Painlevé IV equation. This property in fact holds for an arbitrary real power of the linear factor. Our approach is direct, it is based on an extension of the ladder operators technique developed in [7]. In Section 3 we describe this method, suitable for orthogonal polynomials where the weight has isolated zeros, in particular we derive a pair of fundamental compatibility conditions $\left(S_{1}\right)$ and $\left(S_{2}\right)$. In Section 4 we make use of these to generate non-linear difference equations satisfied by the recurrence coefficients. These difference equations when combined with the Toda equations give a PIV equation satisfied by the recurrence coefficients $\alpha_{n}$.

In the cases of the weights arising from degenerate gaussian ensembles having one multiple eigenvalue $t$ of degeneracy $K$ the multiplicity of the linear factor in the weight is $2 K$. In this case the recurrence coefficients are rational as functions of $t$. The theory of rational solutions to PIV [22] results in the expression of the recurrence coefficients through the generalized Hermite polynomials.

We also mention here that the Hankel determinants associated to the Hermite weights perturbed by a factor are related to the Hankel determinants of the Hermite weight with the addition of $\delta$-function and its derivatives [15]. For an alternative derivation using Heine's multiple integral see [8].

\section{$2 \quad$ Non-generic random matrices}

Let $\mathcal{H}_{N}$ be the space of Hermitian matrices of size $N$ and let $m=\left(m_{1}, m_{2}, \ldots, m_{k}\right)$ be a partition of $N$. Consider the (nonlinear) subspace $\mathcal{H}_{N}^{m}$ in $\mathcal{H}_{N}$ consisting of matrices having the eigenvalues with prescribed multiplicities $m_{1}, \ldots, m_{k}$. That is we suppose the spectrum $\left\{\lambda_{1}, \ldots, \lambda_{N}\right\}$ of an arbitrary element $A \in \mathcal{H}_{N}^{m}$ has the multiplicities described below:

$$
\begin{aligned}
\mu_{1} & =\lambda_{1}=\ldots=\lambda_{m_{1}}, \\
\mu_{2} & =\lambda_{m_{1}+1}=\ldots=\lambda_{m_{1}+m_{2}}, \\
& \cdot \\
& \cdot \\
\mu_{k} & =\lambda_{m_{1}+m_{2}+\ldots+m_{k-1}+1}=\ldots=\lambda_{N},
\end{aligned}
$$

where we have renamed the eigenvalues as $\mu_{1}, \ldots, \mu_{k}$ without repetitions.

As every Hermitian matrix $A$ is diagonalizable, we have

$$
A=U \Lambda U^{-1}
$$


where $\Lambda=\operatorname{diag}\left(\lambda_{1}, \ldots, \lambda_{N}\right)$, and $U$ is unitary. The matrix $U$ is constructed out of a certain orthonormal basis where $A$ becomes diagonal. Such a basis is defined up to unitary transformations leaving the eigenspaces invariant. Therefore $U$ is determined as an element of the homogenous space

$$
U \in U(N) / U\left(m_{1}\right) \times \ldots \times U\left(m_{k}\right)
$$

where the direct product $U\left(m_{1}\right) \times \ldots \times U\left(m_{k}\right)$ of unitary matrices of orders $m_{1}, \ldots, m_{k}$ is embedded into $U(N)$ as diagonal block. More precisely, in order to determine $U$ uniquely, we also assume that the eigenvalues $\mu_{i}, \mu_{j}$ having equal multiplicities $m_{i}=m_{j}$ are such that $\mu_{i}<\mu_{j}$ if $i<j$.

Although the subspace $\mathcal{H}_{N}^{m}$, of $\mathcal{H}_{N}$ is a measure zero set, we may nonetheless construct a natural probability measure of the matrices lying in it. The metric

$$
(d s)^{2}=\operatorname{tr}\left(d H^{*} d H\right)
$$

is well-defined in the subspace $\mathcal{H}_{N}^{m}$. Therefore this metric also naturally defines a measure on the subspace $\mathcal{H}_{N}^{m}$, via the Riemann volume formula. It happens, just like in the case of Hermitian matrices with distinct eigenvalues, with the spectral decomposition (2.2), the measure on $\mathcal{H}_{N}^{m}$ is a product of a measure on the eigenvalues and a measure on the homogeneous space (2.3).

Proposition 1 The metric 2.4) restricted to the subspace $\mathcal{H}_{N}^{m}$ has the form

$$
(d s)^{2}=\sum_{i=1}^{k} m_{i} d \mu_{i}^{2}+2 \sum_{1 \leqslant i<j \leqslant k}\left(\mu_{i}-\mu_{j}\right)^{2}\left(d s_{i j}\right)^{2},
$$

where

$$
\left(d s_{i j}\right)^{2}=\sum_{\substack{m_{1}+\ldots+m_{i-1}+1 \leqslant \alpha \leqslant m_{1}+\ldots+m_{i} \\ m_{1}+\ldots+m_{j-1}+1 \leqslant \beta \leqslant m_{1}+\ldots+m_{j}}}\left(U^{-1} d U\right)_{\alpha \beta}\left(\bar{U}^{-1} \overline{d U}\right)_{\alpha \beta} .
$$

The corresponding volume form on $\mathcal{H}_{N}^{m}$ is

$$
d \mu=\prod_{1 \leqslant i<j \leqslant k}\left(\mu_{i}-\mu_{j}\right)^{2 m_{i} m_{j}} \prod_{i=1}^{k} d \mu_{i} d \nu(U)
$$

where $d \nu(U)$ is invariant measure on the homogeneous space (2.3).

Proof. From decomposition (2.2) we obtain

$$
d A=U\left(d \Lambda+U^{*} d U \Lambda-\Lambda U^{*} d U\right) U^{*} .
$$

Then the metric (2.4) can be rewritten as follows:

$$
(d s)^{2}=\operatorname{tr}\left((d \Lambda)^{2}+2(\delta U \Lambda-\Lambda \delta U) d \Lambda+(\Lambda \delta U)^{2}+(\delta U \Lambda)^{2}-2 \delta U \Lambda^{2} \delta U\right)
$$


where $\delta U:=U^{-1} d U$ and we have used the cyclic property of the trace. Simplifying this further we arrive at

$$
(d s)^{2}=\operatorname{tr}\left((d \Lambda)^{2}+2 \sum_{i \neq j}\left(\lambda_{i} \lambda_{j} \delta U_{i j} \delta U_{j i}-\lambda_{i}^{2} \delta U_{i j} \delta U_{j i}\right)\right)
$$

and since $\delta U$ is anti-Hermitian we get (c.f., e.g., [14, [29]) that the above reduces to

$$
(d s)^{2}=\sum_{i=1}^{N}\left(d \lambda_{i}\right)^{2}+2 \sum_{1 \leqslant i<j \leqslant N}\left(\lambda_{i}-\lambda_{j}\right)^{2} \delta U_{i j} \overline{\delta U_{i j}} .
$$

Recalling the degeneracy conditions (2.1) we note that some of the terms vanish and the restricted metric takes the form

$$
(d s)^{2}=\sum_{i=1}^{k} m_{i}\left(d \mu_{i}\right)^{2}+2 \sum_{1 \leqslant i<j \leqslant k}\left(\mu_{i}-\mu_{j}\right)^{2}\left(d s_{i j}\right)^{2},
$$

where $d s_{i j}$ is defined in (2.5). The second sum in (2.7) is well defined in the homogeneous space.

To determine the corresponding measure we fix locally the section of the representatives of the coset classes and consider coordinates $u_{\alpha \beta}$ such that $d u_{\alpha \beta}=\left(U^{-1} d U\right)_{\alpha \beta}$, where the indices $\alpha<\beta$ are such that $(\alpha \beta) \notin \Delta$. Here $\Delta$ is the diagonal block containing $U\left(m_{1}\right) \times \ldots \times U\left(m_{k}\right)$. Such local coordinates $u_{\alpha \beta}$ will exist if the section is chosen to satisfy $\delta U_{i j}=0$ when $(i j) \in \Delta$. Then taking the real and imaginary parts $\Re u_{\alpha \beta}, \Im u_{\alpha \beta}$, as real coordinates the metric (2.7) becomes a diagonal metric $g_{i i}$ and the term $2\left(\mu_{i}-\mu_{j}\right)^{2}$ appears $2 m_{i} m_{j}$ times along the diagonal. From the Riemann volume formula, the measure corresponding to (2.7) is

$$
\prod_{i=1}^{M} \sqrt{\left|g_{i i}\right|} \prod_{i=1}^{k} d \mu_{i} \prod_{\substack{(\alpha \beta) \notin \Delta \\ \alpha<\beta}} d \Re u_{\alpha \beta} d \Im u_{\alpha \beta}
$$

where

$$
\prod_{i=1}^{M} \sqrt{\left|g_{i i}\right|}=\prod_{1 \leq i<j \leq k} 2^{m_{i} m_{j}}\left(\mu_{i}-\mu_{j}\right)^{2 m_{i} m_{j}} \prod_{i=1}^{k} m_{i}^{1 / 2},
$$

and $M=\operatorname{dim} \mathcal{H}_{N}^{m}$. Thus we obtain the result (2.6) with the measure $d \nu(U)$ given by

$$
d \nu(U)=\prod_{i=1}^{k} m_{i}^{1 / 2} \prod_{\substack{(\alpha \beta) \notin \Delta \\ \alpha<\beta}}\left(U^{-1} d U\right)_{\alpha \beta}\left(\bar{U}^{-1} \overline{d U}\right)_{\alpha \beta}
$$

Remark 1. A large class of generalized random matrix ensembles was recently considered in [1] where a formula for joint probability density of the eigenvalues was obtained. Expression (2.6) may be obtained from that work. 
Remark 2. One way to generalize Proposition 1 is to consider real symmetric matrices with multiple spectrum, then angular variables are given by a factor in the orthogonal group. Same arguments as above lead to the following joint probability density of eigenvalues

$$
\prod_{1 \leqslant i<j \leqslant k}\left(\mu_{i}-\mu_{j}\right)^{m_{i} m_{j}} \prod_{i=1}^{k} d \mu_{i}
$$

Another possibility is to consider degenerate circular ensembles, that is unitary (or other) ensembles with given spectrum multiplicities. In this case the calculation of joint probability density results in taking the appropriate powers of nontrivial Cartan roots.

It is a well-known result of Random Matrix theory [20] that the partition function of any unitary invariant matrix ensemble defined by the multiple integral,

$$
\Delta_{N}[w]:=\frac{1}{N !} \int_{a}^{b} \ldots \int_{a}^{b} \prod_{1 \leq i<j \leq N}\left(x_{i}-x_{j}\right)^{2} \prod_{k=1}^{N} w\left(x_{k}\right) d x_{k}
$$

has the alternative representations, namely

$$
\begin{aligned}
\Delta_{N}[w] & =\operatorname{det}\left(\int_{a}^{b} x^{i+j} w(x) d x\right)_{i, j=0}^{N-1} \\
& =\operatorname{det}\left(\int_{a}^{b} p_{i}(x) p_{j}(x) w(x) d x\right)_{i, j=0}^{N-1},
\end{aligned}
$$

where $p_{l}(x)$ is an arbitrary monic polynomials of exact degree $l$. Now if we orthogonalise these with respect to the weight $w$ over $[a, b]$, namely,

$$
\int_{a}^{b} p_{i}(x) p_{j}(x) w(x) d x=h_{i} \delta_{i, j}
$$

where $h_{i}, i \in \mathbb{N}$ is the square of the $L^{2}$ norm, then (2.8) becomes,

$$
\Delta_{N}[w]=\prod_{j=0}^{N-1} h_{j}
$$

For the generic Gaussian Unitary Ensembles, $w(x)=\exp \left(-x^{2}\right), x \in \mathbb{R}$. In the case of a single degenerate eigenvalue $t$ with $K$ fold degeneracy and the rest, $n$ eigenvalues are distinct, such that $N=n+K$, we find, by relabeling, $\mu_{1}=t, \mu_{2}=x_{1}, \ldots, \mu_{k}=x_{n}$, the partition function reads,

$$
\Delta_{n+K}=\int_{-\infty}^{\infty} \mathrm{e}^{-K t^{2}} D_{n}(t) d t
$$

where

$$
D_{n}(t)=\frac{1}{n !} \int_{-\infty}^{\infty} . . \int_{-\infty}^{\infty} \prod_{1 \leq i<j \leq n}\left(x_{i}-x_{j}\right)^{2} \prod_{l=1}^{n}\left(x_{l}-t\right)^{2 K} \mathrm{e}^{-x_{l}^{2}} d x_{l} .
$$


We note the partition function expressions (2.12), (2.8) are defined here up to constant multiples that come from the integration over the corresponding homogeneous spaces.

The weight of orthogonal polynomials associated with integral (2.13) is the Hermite weight multiplied by an isolated zero, that is,

$$
w(x ; t)=\exp \left(-x^{2}\right)|x-t|^{2 K}, \quad x, t \in \mathbb{R} .
$$

Other crucial characteristics of Random Matrix ensembles are the correlation functions of the eigenvalues. These are obtained by calculating the partition function type integrals (2.8) when some of the eigenvalues are fixed. In the case of single degenerate eigenvalue those correlation functions that involve the multiple eigenvalue coincide with the averages of the powers of characteristic polynomial for the appropriate standard Gaussian unitary ensemble, as it is immediately seen from (2.12)-(2.13). These averages were obtained in the determinant form in [5].

\section{Ladder operators}

We now develop a differentiation formula for the polynomials $p_{n}(x)$ orthogonal with respect to the weight $w_{0}(x)|x-t|^{\gamma}$ on the real line, for any smooth reference weight $w_{0}$ and for general $\gamma \geq 0$. The derivation given here is similar to what was previously known [7, 9], but adapted to the situation where the weight vanishes at one point.

From the orthogonality condition, there follows the recurrence relations;

$$
z p_{n}(z)=p_{n+1}(z)+\alpha_{n} p_{n}(z)+\beta_{n} p_{n-1}(z)
$$

with the initial conditions $p_{0}(z)=1$, and $\beta_{0} p_{-1}(z)=0$. The diagonal recurrence coefficients can then be expressed as

$$
\alpha_{n}=\mathrm{p}_{1}(n)-\mathrm{p}_{1}(n+1)
$$

where $\mathrm{p}_{1}(n)$ are defined by expansions

$$
p_{n}(z)=z^{n}+\mathrm{p}_{1}(n) z^{n-1}+\ldots
$$

The coefficients of the orthogonal polynomials will also have $t$ dependence due to the $t$ dependence of the weight although we denote the polynomials as $p_{n}(z)$.

Since $p_{n}(z)$ is a polynomial of degree $n$, its derivative is a polynomial of degree $n-1$ and can therefore be expressed as a linear combination of $p_{k}(z), \quad k=0,1, \ldots, n-1$, namely,

$$
p_{n}^{\prime}(z)=\sum_{k=0}^{n-1} C_{n, k} p_{k}(z) .
$$

To determine the coefficients $C_{n, k}$ we use orthogonality relations and the formula

$$
\partial_{x}|x-t|^{\gamma}=\delta(x-t)\left((x-t)^{\gamma}-(t-x)^{\gamma}\right)+\gamma \frac{|x-t|^{\gamma}}{x-t} .
$$


We have

$$
\begin{aligned}
C_{n, k}= & \frac{1}{h_{k}} \int_{-\infty}^{\infty} p_{n}^{\prime}(y) p_{k}(y) w_{0}(y)|y-t|^{\gamma} d y \\
= & -\frac{1}{h_{k}} \int_{-\infty}^{\infty} p_{n}(y) p_{k}(y)\left(w_{0}^{\prime}(y)|y-t|^{\gamma}+w_{0}(y) \partial_{y}|y-t|^{\gamma}\right) d y \\
= & -\frac{1}{h_{k}} \int_{-\infty}^{\infty} p_{n}(y) p_{k}(y)\left(\mathrm{v}_{0}^{\prime}(z)-\mathrm{v}_{0}^{\prime}(y)\right) w(y, t) d y \\
& -\frac{\gamma}{h_{k}} \int_{-\infty}^{\infty} p_{n}(y) p_{k}(y) w_{0}(y) \frac{|y-t|^{\gamma}}{y-t} d y \\
= & -\frac{1}{h_{k}} \int_{-\infty}^{\infty} p_{n}(y) p_{k}(y)\left(\mathrm{v}_{0}^{\prime}(z)-\mathrm{v}_{0}^{\prime}(y)\right) w(y, t) d y \\
& -\frac{\gamma}{h_{k}} \int_{-\infty}^{\infty} \frac{p_{n}(y) p_{k}(y)}{y-t} w(y, t) d y
\end{aligned}
$$

where we used notation $\mathrm{v}_{0}(z)=-\log w_{0}(z)$.

We note that analogous consideration of $C_{n, n}=0$ implies

$$
\int_{-\infty}^{\infty} p_{n}^{2}(y) \mathrm{v}_{0}^{\prime}(y) w(y, t) d y=\gamma \int_{-\infty}^{\infty} \frac{p_{n}^{2}(y)}{y-t} w(y, t) d y
$$

also the property $C_{n, n-1}=n$ implies the following Freud equation

$$
n=\frac{1}{h_{n-1}} \int_{-\infty}^{\infty} p_{n}(y) p_{n-1}(y) \mathrm{v}_{0}^{\prime}(y) w(y, t) d y-\frac{\gamma}{h_{n-1}} \int_{-\infty}^{\infty} \frac{p_{n}(y) p_{n-1}(y)}{y-t} w(y, t) d y .
$$

Substitution of $C_{n, k}$ into (3.3) and summation over $k$ using the Christoffel-Darboux formula;

$$
\sum_{j=0}^{n-1} \frac{p_{j}(x) p_{j}(y)}{h_{j}}=\frac{p_{n}(x) p_{n-1}(y)-p_{n}(y) p_{n-1}(x)}{h_{n-1}(x-y)}
$$

produces the differentiation formula;

$$
p_{n}^{\prime}(z)=-B_{n}(z) p_{n}(z)+\beta_{n} A_{n}(z) p_{n-1}(z),
$$

where

$$
\begin{aligned}
A_{n}(z) & :=\frac{1}{h_{n}} \int_{-\infty}^{\infty} \frac{\mathrm{v}_{0}^{\prime}(z)-\mathrm{v}_{0}^{\prime}(y)}{z-y} p_{n}^{2}(y) w(y, t) d y+a_{n}(z, t) \\
a_{n}(z, t) & :=\frac{\gamma}{h_{n}} \int_{-\infty}^{\infty} \frac{p_{n}^{2}(y)}{(y-t)(z-y)} w(y, t) d y \\
B_{n}(z) & :=\frac{1}{h_{n-1}} \int_{-\infty}^{\infty} \frac{\mathrm{v}_{0}^{\prime}(z)-\mathrm{v}_{0}^{\prime}(y)}{z-y} p_{n}(y) p_{n-1}(y) w(y, t) d y+b_{n}(z, t) \\
b_{n}(z, t) & :=\frac{\gamma}{h_{n-1}} \int_{-\infty}^{\infty} \frac{p_{n}(y) p_{n-1}(y)}{(y-t)(z-y)} w(y, t) d y
\end{aligned}
$$


Equation (3.8) is the "lowering" operator.

A direct calculation produces two fundamental compatibility conditions valid for all $z$;

$$
\begin{gathered}
B_{n+1}(z)+B_{n}(z)=\left(z-\alpha_{n}\right) A_{n}(z)-\mathrm{v}_{0}^{\prime}(z) \\
1+\left(z-\alpha_{n}\right)\left(B_{n+1}(z)-B_{n}(z)\right)=\beta_{n+1} A_{n+1}(z)-\beta_{n} A_{n-1}(z) .
\end{gathered}
$$

where we have used (3.6) to arrive at $\left(S_{1}\right)$. Without going into details, we mention here that if the factor $|x-t|^{\gamma}$ in the weight $w(x, t)$ is replaced by

$$
\prod_{j=1}^{\mathcal{N}}\left|x-t_{j}\right|^{\gamma_{j}}
$$

then $\left(S_{1}\right)$ and $\left(S_{2}\right)$ still hold and the only changes are

$$
\begin{aligned}
a_{n}\left(z, t_{1}, . ., t_{\mathcal{N}}\right) & =\sum_{j=1}^{\mathcal{N}} \frac{\gamma_{j}}{h_{n}} \int_{-\infty}^{\infty} \frac{p_{n}^{2}(y)}{\left(y-t_{j}\right)(z-y)} w\left(y, t_{1}, . ., t_{\mathcal{N}}\right) d y \\
b_{n}\left(z, t_{1}, . ., t_{\mathcal{N}}\right) & =\sum_{j=1}^{\mathcal{N}} \frac{\gamma_{j}}{h_{n-1}} \int_{-\infty}^{\infty} \frac{p_{n}(y) p_{n-1}(y)}{\left(y-t_{j}\right)(z-y)} w\left(y, t_{1}, . ., t_{\mathcal{N}}\right) d y .
\end{aligned}
$$

Using $\left(S_{1}\right)$ and recurrence relations we have the "raising" operator,

$$
p_{n-1}^{\prime}(z)=\left(B_{n}(z)+\mathrm{v}_{0}^{\prime}(z)\right) p_{n-1}(z)-A_{n-1}(z) p_{n}(z)
$$

In the next section we take $w_{0}(x)=\exp \left(-x^{2}\right)$, and make use of $\left(S_{1}\right)$ and $\left(S_{2}\right)$ to produce a pair of non-linear difference equations satisfied by the recurrence coefficients for fixed $t$. These when combined with the $t$ - evolution equations satisfied by the recurrence coefficients result in a particular Painlevé IV.

\section{Derivation of the Painlevé equation}

For $w(x, t)=\exp \left(-x^{2}\right)|x-t|^{\gamma}, \mathrm{v}_{0}(x)=x^{2}$, we find,

$$
\begin{aligned}
& A_{n}(z)=2+a_{n}(z, t) \\
& B_{n}(z)=b_{n}(z, t) .
\end{aligned}
$$

For $z$ near $\infty$, with fixed $t$, we obtain the following asymptotic expansions;

$$
\begin{aligned}
& a_{n}(z, t) \sim \frac{2 \alpha_{n}}{z}+\frac{\gamma+2 t \alpha_{n}}{z^{2}}+\frac{\gamma t+\gamma \alpha_{n}+2 t^{2} \alpha_{n}}{z^{3}}+. . \\
& b_{n}(z, t) \sim \frac{2 \beta_{n}-n}{z}+\frac{t\left(2 \beta_{n}-n\right)}{z^{2}}+\frac{\gamma \beta_{n}+t^{2}\left(2 \beta_{n}-n\right)}{z^{3}}+. .
\end{aligned}
$$


where the coefficients are determined from orthogonality, the recurrence relations, (3.6) and (3.7).

Substituting the asymptotic expansions into $\left(S_{1}\right)$ and $\left(S_{2}\right)$, we find, by comparing the coefficients of $1 / z^{j}$, two difference equations satisfied by $\alpha_{n}$ and $\beta_{n}$;

$$
\begin{gathered}
\beta_{n+1}+\beta_{n}=n+\frac{1}{2}+\frac{\gamma}{2}+\alpha_{n}\left(t-\alpha_{n}\right) \\
\left(t-\alpha_{n}\right)\left(\beta_{n+1}-\beta_{n}-\frac{1}{2}\right)=\beta_{n+1} \alpha_{n+1}-\beta_{n} \alpha_{n-1} .
\end{gathered}
$$

Remark 1. If $\gamma=0$, then $\alpha_{n}=0$, thus (4.5) and (4.6) become $\beta_{n+1}+\beta_{n}=n+1 / 2$ and $\beta_{n+1}-\beta_{n}=1 / 2$ respectively. The solution of these equations, subject to the initial condition $\beta_{0}=0$ is $\beta_{n}=n / 2$, which is the recurrence coefficients of the Hermite polynomials.

Remark 2. If $t=0$, then $\alpha_{n}=0$, then (4.5) becomes $\beta_{n+1}+\beta_{n}=n+(1+\gamma) / 2$. The unique solution subject to the initial condition $\beta_{0}=0$ is $\beta_{n}=n / 2+\gamma\left(1-(-1)^{n}\right) / 4$, which is the recurrence coefficient of what Szegö called the generalized Hermite polynomials (see [27, problem 25). These should not be confused with the generalized Hermite polynomials which arise in the rational solutions of Painlevé IV (see next section).

To study the $t$-evolution of the recurrence coefficients we begin by taking a derivative with respect to $t$ of the squared norm $h_{n}$ of the $n$-th orthogonal polynomial,

$$
\frac{\partial_{t} h_{n}}{h_{n}}=\frac{\gamma}{h_{n}} \int_{-\infty}^{\infty} \frac{p_{n}^{2}(y)}{t-y} w(y, t) d y=-2 \alpha_{n},
$$

where the last equality is obtained by using relation (3.6) and noting that $\mathrm{v}_{0}^{\prime}(y)=2 y$. Since $\beta_{n}=h_{n} / h_{n-1}$, equation (4.7) implies

$$
\frac{\partial_{t} \beta_{n}}{\beta_{n}}=2\left(\alpha_{n-1}-\alpha_{n}\right)
$$

Differentiating relation

$$
0=\int_{-\infty}^{\infty} p_{n}(y) p_{n-1}(y) w(y, t) d y
$$

with respect to $t$, we find,

$$
0=h_{n-1} \partial_{t} \mathrm{p}_{1}(n)+\int_{-\infty}^{\infty} p_{n}(y) p_{n-1}(y) w_{0}(y) \partial_{t}|y-t|^{\gamma} d y
$$

where function $\mathrm{p}_{1}(n)$ was defined in (3.2). Using the Freud equation (3.7) we now get

$$
\begin{aligned}
\partial_{t} \mathrm{p}_{1}(n) & =\frac{\gamma}{h_{n-1}} \int_{-\infty}^{\infty} \frac{p_{n}(y) p_{n-1}(y)}{y-t} w(y, t) d y \\
& =\frac{2}{h_{n-1}} \int_{-\infty}^{\infty} y p_{n}(y) p_{n-1}(y) w(y, t) d y-n=2 \beta_{n}-n
\end{aligned}
$$


In view of relation (3.1),

$$
\partial_{t} \alpha_{n}=2\left(\beta_{n}-\beta_{n+1}\right)+1 .
$$

The equations (4.8) and (4.10) are the Toda evolution equations.

We now show that $\widetilde{D}_{n}:=D_{n} \exp \left(n t^{2}\right)$ satisfies the Toda molecule equation (c.f. [23]). First note that

$$
\sum_{j=0}^{n-1} \alpha_{j}=-\mathrm{p}_{1}(n) \quad \text { and } \quad \beta_{n}=\frac{D_{n+1} D_{n-1}}{D_{n}^{2}} .
$$

The equation (4.7) together with (4.9) implies

$$
\partial_{t}^{2} \log D_{n}=4 \frac{D_{n+1} D_{n-1}}{D_{n}^{2}}-2 n,
$$

and hence

$$
\partial_{t}^{2} \log \widetilde{D}_{n}=4 \frac{\widetilde{D}_{n+1} \widetilde{D}_{n-1}}{\widetilde{D}_{n}^{2}},
$$

which is the Toda molecule equation.

To proceed further, we parameterize $\beta_{n}$ as

$$
\beta_{n}=\frac{n}{2}+\frac{r_{n}}{2}+\frac{\gamma}{4}, \quad r_{0}=-\frac{\gamma}{2},
$$

then relation (4.5) becomes

$$
\frac{r_{n+1}+r_{n}}{2}=\left(t-\alpha_{n}\right) \alpha_{n} .
$$

Multiplying relation (4.6) by $\alpha_{n}$ and using the previous relation we get

$$
\frac{r_{n+1}^{2}-r_{n}^{2}}{4}=\alpha_{n} \alpha_{n+1} \beta_{n+1}-\alpha_{n} \alpha_{n-1} \beta_{n} .
$$

Therefore

$$
\frac{r_{n}^{2}}{4}=\alpha_{n} \alpha_{n-1}\left(\frac{n}{2}+\frac{r_{n}}{2}+\frac{\gamma}{4}\right)+\mathrm{a},
$$

where a does not depend on $n$. Taking into account the initial condition $r_{0}=-\frac{\gamma}{2}$ we obtain the equation

$$
r_{n}^{2}=2\left(n+r_{n}+\frac{\gamma}{2}\right) \alpha_{n} \alpha_{n-1}+\frac{\gamma^{2}}{4} .
$$

In terms of the variables $r_{n}$, the Toda equations (4.8), (4.10) become

$$
\alpha_{n-1}=\alpha_{n}+\frac{1}{2\left(n+r_{n}+\frac{\gamma}{2}\right)} \partial_{t} r_{n},
$$

and

$$
\partial_{t} \alpha_{n}=r_{n}-r_{n+1}
$$


respectively. Eliminating $r_{n+1}$ from equations (4.15) and (4.12) produces

$$
r_{n}=\alpha_{n}\left(t-\alpha_{n}\right)+\frac{1}{2} \partial_{t} \alpha_{n}
$$

To get the differential equation on $\alpha_{n}$ we substitute expressions (4.14), (4.16) into (4.13):

$$
\left(\alpha_{n}\left(t-\alpha_{n}\right)+\frac{\alpha_{n}^{\prime}}{2}\right)^{2}=\left(2 n+2 \alpha_{n}\left(t-\alpha_{n}\right)+\alpha_{n}^{\prime}+\gamma\right) \alpha_{n}^{2}+\alpha_{n}\left(\alpha_{n}\left(t-\alpha_{n}\right)+\frac{\alpha_{n}^{\prime}}{2}\right)^{\prime}+\frac{\gamma^{2}}{4} .
$$

After simplification we obtain the following result.

Theorem 1 The recurrent coefficients $\alpha_{n}(t)$ satisfy

$$
\alpha_{n}^{\prime \prime}=\frac{\alpha_{n}^{\prime 2}}{2 \alpha_{n}}+6 \alpha_{n}^{3}-8 t \alpha_{n}^{2}+2\left(t^{2}-\gamma-2 n-1\right) \alpha_{n}-\frac{\gamma^{2}}{2 \alpha_{n}}
$$

which is a particular fourth Painlevé equation.

\section{$5 \quad$ Explicit solutions for even multiplicity}

Painlevé IV equation was first represented as a simple system of three first order equations (dressing chain) in [28. Such a symmetric form of PIV was used in 22] to obtain all the rational solutions of the equation in the remarkable determinant form (simultaneously with the independent work [16]). We use the notations from Noumi-Yamada 22] to recall their results and then to use them.

Firstly we bring equation (4.17) to the canonical form by a simple change of variable. Let $y=2 \alpha_{n}$ and $\widetilde{t}=-t$ then (4.17) takes the form

$$
y^{\prime \prime}(\widetilde{t})=\frac{y^{\prime 2}}{2 y}+\frac{3}{2} y^{3}+4 \widetilde{t} y^{2}+2\left(\widetilde{t}^{2}-a\right) y+\frac{b}{y},
$$

where $a=2 n+1+\gamma, b=-2 \gamma^{2}$. Then the symmetric form of PIV is a system of first order differential equations satisfied by $f_{0}=f_{0}(x), f_{1}=f_{1}(x), f_{2}=f_{2}(x)$, where

$$
f_{1}(x)=-c y(-c x)
$$

with $c=\sqrt{-3 / 2}$. The system reads as follows:

$$
\begin{aligned}
f_{0}^{\prime}+f_{0}\left(f_{1}-f_{2}\right) & =b_{0} \\
f_{1}^{\prime}+f_{1}\left(f_{2}-f_{0}\right) & =b_{1} \\
f_{2}^{\prime}+f_{2}\left(f_{0}-f_{1}\right) & =b_{2}
\end{aligned}
$$

where

$$
\begin{aligned}
f_{0}+f_{1}+f_{2} & =3 x \\
b_{0}+b_{1}+b_{2} & =3,
\end{aligned}
$$


and parameters of the PIV are suitably expressed in terms of $b_{0}, b_{1}$ and $b_{2}$. The PIV equation can also be written in the bilinear form on the level of $\tau$-functions. The solution of (4.17) may then be expressed in terms of $\tau$-functions $\tau_{0}(x), \tau_{1}(x), \tau_{2}(x)$ as

$$
f_{1}=\frac{d}{d x} \log \frac{\tau_{2}}{\tau_{0}}+x
$$

where the functions $\tau_{0}$ and $\tau_{2}$ will be defined later.

The generalised Hermite polynomials [22] are defined as

$$
H_{m, n}(x)=\operatorname{det}\left(P_{n-i+j}(x)\right)_{i, j=1}^{m}
$$

where

$$
P_{s}(x)=\sum_{i+2 j=s} \frac{1}{6^{j} i ! j !} x^{i}
$$

They coincide with the specialization $S_{n^{m}}\left(x, \frac{1}{6}, 0,0, \ldots\right)$ of Schur polynomials corresponding to rectangular Young diagrams containing $m$ rows of length $n$.

Define also the set of functions

$$
u_{m, n}(x)=\exp \left(-\frac{x^{4}}{12}+\frac{m-n}{2} x^{2}\right) H_{m, n}(x) .
$$

Then the triple

$$
\left(\tau_{0}, \tau_{1}, \tau_{2}\right)=\left(u_{m, n}, u_{m+1, n}, u_{m, n+1}\right)
$$

leads to a solution of PIV through formulas (5.1) -(5.8) in the case $\gamma=m$.

Theorem 2 The recurrent coefficients $\alpha_{n}$ for the weight $w(x)=e^{-x^{2}}(x-t)^{2 K}$ with $K \in \mathbb{Z}_{+}$ are given by

$$
\alpha_{n}(t)=-\frac{1}{2} \frac{d}{d t} \log \frac{H_{2 K, n+1}(t / c)}{H_{2 K, n}(t / c)}
$$

where $H_{m, n}(x)$ are defined by (5.9), and $c=\sqrt{-3 / 2}$.

Proof. For $\gamma=2 K$ with $K \in \mathbb{N}$ the orthogonal polynomials with the weight $w(x, t)$ can be expressed in terms of Hermite polynomials by the Christoffel formula ([27], pg. 30), since $w(x, t)$ is the Hermite weight multiplied by a polynomials in $x$. It follows from the formula that the recurrence coefficients $\alpha_{n}, \beta_{n}$ are rational functions of $t$. Therefore $\alpha_{n}(t)$ is a rational solution of equation (5.1) in this case. The rational solution of the PIV equation is unique if it exists (see [17]) and is expressed in terms of the generalized Hermite polynomials:

$$
\alpha_{n}(t)=\frac{1}{2} y(-t)=-\frac{1}{2 c} f_{1}\left(\frac{t}{c}\right)=-\frac{1}{2} \frac{d}{d t} \log \frac{H_{2 K, n+1}(t / c)}{H_{2 K, n}(t / c)} .
$$


Remark. There is another way to see rationality of $\alpha_{n}(t)$. Indeed, equation (2.13) defines an even polynomials of degree $2 K n$ in $t$, hence, $h_{n}(t)=D_{n+1}(t) / D_{n}(t)$, is rational in $t$ and (4.7) shows that $\alpha_{n}(t)$ is also rational in $t$.

The above considerations allow us to obtain an expression for the Hankel determinant. We have seen that (4.7),

Therefore

$$
\alpha_{i}=-\frac{\partial_{t} h_{i}}{2 h_{i}}
$$

$$
\log h_{i}-\log \frac{H_{2 K, i+1}(t / c)}{H_{2 K, i}(t / c)}=\text { const. }
$$

So

$$
h_{i}=a_{i} \frac{H_{2 K, i+1}(t / c)}{H_{2 K, i}(t / c)}
$$

for some constant $a_{i}$.

Proposition 2 (c.f. [5], [13]) The Hankel determinant for the weight $w(x)=e^{-x^{2}}(x-t)^{2 K}$ with $K \in \mathbb{Z}_{+}$is given by

$$
D_{n}=A_{K, n} H_{2 K, n}(t / c)
$$

where $c=\sqrt{-3 / 2}$, and

$$
A_{K, n}=\prod_{i=0}^{n-1} a_{i}=(-1)^{K n} \pi^{\frac{n}{2}} \frac{3^{K n} G(2 K+n+1)}{2^{K n+\frac{n(n-1)}{2}} G(2 K+1)}
$$

with the Barnes $G$-function [3] defined by

$$
G(z+1)=\Gamma(z) G(z), \quad G(1)=1 .
$$

Proof. It is clear from (5.11) and the product expression of $D_{n}$,

$$
D_{n}=h_{0} h_{1} \ldots h_{n-1}
$$

where $h_{i}$ are the square of the $L^{2}$ norm of the monic orthogonal polynomials, that the constant $A_{K, n}$ in the proposition depends only on $n, K$, so all we need to do is to determine its value.

Note that the coefficient of $t^{2 K n}$ of $D_{n}(t)$ is equal to the Hankel determinant associated with the Hermite weight. Therefore

$$
D_{n}(t)=t^{2 K n} \pi^{\frac{n}{2}} \prod_{i=0}^{n-1} \frac{i !}{2^{i}}+\text { lower order terms. }
$$

On the other hand the leading coefficient of $H_{2 K, n}(t)$ is equal to

$$
\frac{G(2 K+1) G(n+1)}{G(2 K+n+1)}
$$


(see [22]). Combining (5.12) and (5.13) together we get the value of $A_{K, n}$ as stated.

Remark. The Hankel determinant $D_{n}$ as the average of characteristic polynomial (2.13) was first computed by Brezin and Hikami in [5] as determinant of Hermite polynomials. The equivalence of the resulting formulas with the formulas for the $\tau$-functions $H_{2 K, n}$ for PIV from [22] was used by Forrester and Witte in [13] (see also [16]). We have now an explanation for this coincidence through showing that the diagonal recurrence coefficients $\alpha_{n}(t)$ is a solution of PIV. We also note that this result can be obtained other way round using (4.7) and [13].

\section{Acknowledgements}

M.F. is grateful to A.Borodin and A.P.Veselov for useful discussions. We would like to acknowledge the support of European research programme ENIGMA (contract MRTN-CT2004-5652). M.F. also acknowledges the support of Chapman Fellowship at the Mathematics Department of Imperial College.

\section{References}

[1] An J., Wang Z., Yan K A Generalization of Random Matrix Ensemble I, II math-ph/0502020, math-ph/0502021

[2] Baik J., Deift P., Strahov E. Products and ratios of characteristic polynomials of random Hermitian matricies, J. Math. Phys. 44 (2003), no. 8, 3657-3670.

[3] Barnes E. W. The theory of the G-function, Q.J. Pure Appl. Math. 31(1900)264.

[4] Borodin A., Strahov E. Averages of characteristic polynomials in random matrix theory, Comm. Pure Appl. Math. 59 (2006), no. 2, 161-253.

[5] Brezin E.B. and Hikami S.B. Characteristic polynomials of random matrices, Commun. Math. Phys. 214(2000)113-135.

[6] Chalykh, O., Feigin, M., Veselov, A. New integrable generalizations of Calogero-Moser quantum problem, J. Math. Phys. 39 (1998), no. 2, 695-703.

[7] Chen Y. and Ismail M. Ladder operators and differential equations for orthogonal polynomials, J. Phys. A: Math. Gen. 30(1997)7817-7829.

[8] Chen Y. and Griffin J. Krall type polynomials via Heine formula, J. Phys. A:Math. Gen. 35(2002)637-656.

[9] Chen Y. and Ismail M. Jacobi polynomials from campatibilty conditions, Proc. Amer. Math. Soc. 133(2005)465-472.

[10] Chen Y. and Pruessner G. Orthogonal polynomials with discontinuous weights, J. Phys. A:Math. Gen. 38(2005)L191-198. 
[11] Dueñez E. Random matrix ensembles associated to compact symmetric spaces, Commun. Math. Phys. 244(2004)29-61.

[12] Forrester P. J. Some multidimensional integrals related to many-body systems with the 1/ $r^{2}$ potential, J. Phys. A 25 (1992), no. 10, 607-614.

[13] Forrester P. J. and Witte N. Application of the $\tau$-function theory of Painlevé equations to random matrices: PIV, PII and the GUE, Commun. Math. Phys. 219(2001)357-398.

[14] Di Francesco, P., Ginsparg, P., Zinn-Justin, J. 2 D gravity and random matrices. Phys. Rep. 254 (1995), no. 1-2, 133 pp.

[15] Grünbaum F. A. and Haine L. Orthogonal polynomials satisfying differential equations: the role of the Darboux transformations, CRM proc. Lecture Notes 9 (1996) 143; Grünbaum F. A., Haine L. and Horozov E. Some function that generalize the Krall-Laguerre polynomials, J. Comput. Appl. Math. 106(1999)271.

[16] Kajiwara K., Ohta Y. Determinant structure of the rational solutions for the Painlevé IV equation, J. Phys. A 31 (1998), no. 10 , 2431-2446.

[17] Lukashevich N. A. Theory of the fourth Painlevé equation, Diff. Eq. 3(1967)395-399; Gromak V. I. Theory of the fourth Painlevé equation, 23(1987)506-513; Murata Y. Rational solutions of the second and and the fourth Painlevé equation, Funkcial. Ekvac. 28(1985)1-32

[18] Magnus A.P.Painlevé-type differential equations for the recurrence coefficients of semiclassical orthogonal polynomials, J. Comp. Appl. Math. 57(1995)215-237.

[19] Magnus A.P. Asymptotics for the simplest generalized Jacobi polynomials recurence coefficients from Freud's equations:numerical exploration, Ann.Numer.Math 2 (1995), no. $1-4,311-325$.

[20] Mehta M. L. Random Matrices, third edition, Elsevier, 2004.

[21] Nevai P., Erdélyi T. and Magnus A. P. Generalized Jacobi Weights, Christoffel functions and Jacobi polynomials, SIAM J. Math. Anal. 25(1994)602-614.

[22] Noumi M. and Yamada Y. Symmetries in the fourth Painlevé equation and Okamoto polynomials, Nagoya Math. J. 153(1999)53-86

[23] Okamoto, K. Studies on the Painlev equations. III. Second and fourth Painlevé equations, $P_{\mathrm{II}}$ and $P_{\mathrm{IV}}$. Math. Ann. 275 (1986), no. 2, 221-255.

[24] Sergeev, A. N., Veselov, A.P. Deformed quantum Calogero-Moser problems and Lie superalgebras, Comm. Math. Phys. 245 (2004), no. 2, 249-278. 
[25] Sergeev, A. N., Veselov, A.P. Generalised discriminants, deformed Calogero-MoserSutherland operators and super-Jack polynomials, Adv. Math. 192 (2005), no. 2, 341375.

[26] Snaith N. Derivatives of random matrix characteristic polynomials with applications to elliptic curves J. Phys. A 38 (2005), no. 48, 10345-10360.

[27] G. Szegö, Orthogonal polynomials, Amer. Math. Soc. Colloq. Publ. vol. 23, Providence, R.I., 1975.

[28] Veselov A. P. and Shabat A.B. A dressing chain and the spectral theory of the Schrödinger operator, Funct. Anal. Appl. 27(1993)81-96.

[29] Weyl H. The Classical Groups: Their Invariants and Representation, Princeton University Press, 1997.

[30] Wigner E.P. Random matrices in physics, SIAM Review 9 (1967) 1-23. 